\title{
A theoretical analysis of river bars stability under changing channel width
}

\author{
S. Zen, G. Zolezzi, and M. Tubino \\ Department of Civil, Environmental and Mechanical Engineering, via Mesiano 77, 38123, Trento, Italy
}

Correspondence to: S. Zen (simone.zen@unitn.it)

Received: 15 October 2013 - Revised: 3 February 2014 - Accepted: 14 February 2014 - Published: 1 April 2014

\begin{abstract}
In this paper we propose a new theoretical model to investigate the influence of temporal changes in channel width on river bar stability. This is achieved by performing a nonlinear stability analysis, which includes temporal width variations as a small-amplitude perturbation of the basic flow. In order to quantify width variability, channel width is related with the instantaneous discharge using existing empirical formulae proposed for channels with cohesionless banks. Therefore, width can vary (increase and/or decrease) either because it adapts to the temporally varying discharge or, if discharge is constant, through a relaxation relation describing widening of an initially overnarrow channel towards the equilibrium width. Unsteadiness related with changes in channel width is found to directly affect the instantaneous bar growth rate, depending on the conditions under which the widening process occurs. The governing mathematical system is solved by means of a two-parameters $(\epsilon, \delta)$ perturbation expansion, where $\epsilon$ is related to bar amplitude and $\delta$ to the temporal width variability. In general width unsteadiness is predicted to play a destabilizing role on free bar stability, namely during the peak stage of a flood event in a laterally unconfined channel and invariably for overnarrow channels fed with steady discharge. In this latter case, width unsteadiness tends to shorten the most unstable bar wavelength compared to the case with constant width, in qualitative agreement with existing experimental observations.
\end{abstract}

\section{Introduction}

River bars have been extensively studied, analytically, as an instability phenomenon of an incompressible flow over a flat mobile bed in a single-thread river channels. Practical reasons of this interest lays in the need to predict bars formation in channelized rivers and the related scour and deposition processes that can affect navigation or damage engineering structures (e.g. bank protection).

Most of these bar theories have been developed under simplifying assumptions of steady discharge and fully sediment transporting cross section, therefore their application is strictly suitable mostly for single-thread channels and alternate bar patterns. Despite many simplifying assumptions, bar theories have been effective in supporting quantitative understanding of bar processes (e.g. conditions of occurrence; lenght scales) and have received quantitative support from laboratory experiments. Despite their simplifying assumptions, their application has been extended to be used as physically-based predictors of alluvial channel pattern (e.g. Parker, 1976; Crosato and Mosselman, 2009) with some degree of success.

Moreover evidence has been reported that rather simple and regular bar dynamics can take place also in complex channel morphologies, as wandering (Church and Rice, 2009) or braiding (Zolezzi et al., 2012). In this latter study the formation and downstream migration of alternate and central bar patterns has been observed in a main individual branch of a braided river during a bar-forming flood event below bankfull stage. In such case, the channel transporting sediments expands its width during the rising stage of the flood, and is afterwards contracted during the falling limb. Application of the classical bar theory using constant discharge and channel width values, averaged over the flood duration, yields several discrepancies in bar wavelength and dominant transverse modes.

In principle, however, bar theory can be applied to laterally unconfined channels, provided temporal variations of discharge and actively transporting channel width are properly accounted for. 
The aim of the present work is therefore to investigate, on a modelling basis, the stability of river bars in channels where the active width changes with time, thus possibly affecting bar morphodynamics. Because the channel width-todepth ratio plays a key role on bar development, its temporal variations associated with the widening process can be expected to affect the dynamics of contemporarily developing bars. Existing theoretical analyses (e.g. Repetto et al., 2002) have investigated the influence of spatial planform variability on bars formation showing how spatial changes in channel width, influencing river bars, may produce planform instability and a related tendency to braid. The role of width unsteadiness may become relevant especially in laterally unconfined channels with non-cohesive banks, as it has been observed in laboratory experiments on the initiation of braided and of "pseudo-meandering" streams (Ashmore, 1982, 1991; Bertoldi and Tubino, 2005; Visconti et al., 2010). Evidence of this dynamics has been provided, also, by field observation on an artificially re-shaped natural river consequentially to a series of flood events (Lewin, 1976); this highlights the mutual influence between planform and bar instability in streams where the evolution of bed and banks occur at comparable time scales. Despite the fact that unsteadiness is ubiquitous in natural river systems, only very few theoretical analyses have addressed the role of flow unsteadiness on bars formation (Tubino, 1991; Hall, 2004) while an analysis of the role of width unsteadiness is even lacking. Overall, the present work is therefore expected to contribute a novel theoretical understanding about the applicability of analytical bar theories to real river systems.

\section{Methods}

\subsection{Conceptual approach}

In order to understand the impact of width unsteadiness on bar stability in a straight channel, we first need to characterize the processes that lead to temporal changes in the active channel width. Their mathematical description will then be included in existing approaches for classical bar stability analyses, allowing to build the desired model. Four main hydromorphological configurations, based on different combinations of channel width and discharge variability, are examined (Fig. 1): (a) ideal channels with both constant width and discharge, like in most common laboratory flume experiments on river bars; (b) channelized rivers with constant width subject to streamflow variability and often developing alternate bars; (c) constant discharge flowing in an initially overnarrow channel with non cohesive banks, as typical of the initial experimental condition of physical models of braided rivers; and (d) laterally unconfined channels subject to floods, as it can be the case of the main active branches of braided rivers. Configuration (a) is representative of most of the conditions to which classical bar theories strictly ap- ply. Bar dynamics in configuration b) has been investigated only by the theories of Tubino (1991), Hall (2004) and by the field investigations by Welford (1994). The present contribution specifically aims to investigate free bar stability referring to configurations (c) and (d).

\subsection{Quantification of width unsteadiness}

In order to keep the mathematical problem suitable for analytical solutions, simple relationships to express channel width variability are needed. A first, simple attempt has been made by using classical regime formulaes to relate bankfull discharge with channel width in single-thread channels. Such relationships are strictly valid under long-term, equilibrium conditions: therefore the present approach is based on the assumption of an "instantaneous" validity of regime equations. Note that this assumption should be less crude than it may appear, because we are interested here more in a simple mathematical law describing the trends and order of magnitude of width unsteadiness rather than in a predictive formnula quantitatively valid for a specific case. To this aim we use the empirical formula proposed by Ashmore (1982) to predict the width at river equilibrium stage for anabranch channels in braided rivers:

$W^{*}=\alpha_{\mathrm{W}} Q^{* n_{\mathrm{W}}} \quad \alpha_{\mathrm{W}}=8.1019, n_{\mathrm{B}}=0.4738$

In Eq. (1) $W^{*}$ represent the dimensional channel width and $Q^{*}$ the related flow discharge in equilibrium conditions. Hereinafter we indicate with a star $\left(^{*}\right)$ dimensional quantities. According to our purposes relation (Eq. 1) can be read as the equilibrium width to which a laterally unconfined channel tends asimptotically for a given discharge. A suitable dimensionless expression for Eq. (1) has been derived to fit a dimensionless mathematical approach. Such expression is obtained by following the procedure proposed by Parker et al. (2007). In the following relation the dimensionless channel half-width $B$ is also introduced for formal consistency with existing bar theories:

$$
\begin{aligned}
& W=2 B=2 \alpha_{\mathrm{n}} Q^{n_{\mathrm{W}}} \\
& \alpha_{\mathrm{n}}=0.5 \alpha_{\mathrm{W}} F_{0}^{n_{\mathrm{W}}} \bar{\beta}^{n_{\mathrm{W}}-1} \bar{d}_{\mathrm{s}}^{-5 / 2 n_{\mathrm{W}}+1}
\end{aligned}
$$

where the dimensionless parameters are defined as follows:

$$
F_{0}=\frac{U_{0}^{*}}{\sqrt{g D_{0}^{*}}} \quad \bar{\beta}=\frac{B_{0}^{*}}{D_{0}^{*}} \quad \bar{d}_{\mathrm{s}}=\frac{d_{\mathrm{s}}^{*}}{D_{0}^{*}}
$$

with $d_{\mathrm{s}}^{*}$ the median sediment grain size, $B_{0}^{*}$ the reference half-channel width value and $U_{0}^{*}, D_{0}^{*}$ the components of the uniform flow chosen as reference scales.

Two different time variables are considered when examining both cases. The variable $\tau$ will vary on the externally imposed time scale of width and discharge unsteadiness, while the variable $t$ will be used to denote the relevant "intrinsic" time variable for the temporal morphodynamics of bars. 
These two variables will be considered as mutually independent within the present theoretical analysis, while for applications of the theory to specific real cases the related time scales shall be quantified and possible interactions assessed. In general, the externally imposed time scale is quite "fast", i.e. much shorter than that associated with temporal width adjustments that occur in regulated rivers because of landuse changes, gravel mining, dam construction (Surian and Rinaldi, 2003).

\subsubsection{Constant discharge}

This is the case of an initially overnarrow channel fed by constant discharge(Fig. 1c), for which the minimum width for bars formation predicted by the bar theories is larger than the imposed initial width (Bertoldi and Tubino, 2005). To describe the widening process, we simply assume that the erosion occurs homogeneously at the banks and that the channel tends, asimptotically, to achieve the equilibrium width at a widening rate that linearly decreases with the distance from the equilibrium conditions. This simplified law is based on the consideration that the erosion process, increases the channel width and decreases the sediment transport of the flow, reducing, in turn, its ability of entering sediments from the banks and thus slowing down the widening process. This process occurs faster while the initial geometry of the channel is far from equilibrium and reduces its effect progressively approaching the final conditions. Therefore, under this assumption, the relaxation relation (Eq. 5) is proposed whereby the value of channel width $\left(2 B_{\mathrm{E}}^{*}\right)$ at equilibrium with the specified discharge is evaluated through Eq. (2):

$$
\left\{\begin{array}{l}
B^{*}\left(\tau^{*}\right), \tau^{*}=\left(\frac{U^{*}}{B^{*}}\right)_{\mathrm{E}}\left(B_{\mathrm{E}}^{*}-B^{*}\left(\tau^{*}\right)\right) \\
B^{*}\left(\tau^{*}=0\right)=B_{0}^{*} \\
B^{*}\left(\tau^{*} \rightarrow \infty\right)=B_{\mathrm{E}}^{*}
\end{array}\right.
$$

The complete solution for this case reads:

$$
B^{*}\left(\tau^{*}\right)=B_{\mathrm{E}}^{*}+\left(B_{\mathrm{E}}^{*}-B_{0}^{*}\right)\left(-e^{-\alpha \tau^{*}}\right)
$$

which, scaled by the unperturbed half channel width $B_{\mathrm{E}}^{*}$, can be written in its dimensionless form as:

$B(\tau)=\frac{B_{\mathrm{E}}^{*}}{B_{\mathrm{E}}^{*}}+\frac{\left(B_{\mathrm{E}}^{*}-B_{0}^{*}\right)}{B_{\mathrm{E}}^{*}}\left(-e^{-\alpha \tau}\right)$.

In Eq. (7) $\tau$ is a dimensionless time variable defined as

$$
\tau=k \frac{U_{\mathrm{E}}^{*}}{\left(B_{\mathrm{E}}^{*}-B_{0}^{*}\right)} \tau^{*},
$$

where the parameter $k$ accounts for the erodibility of both banks, assumed to be uniform in space.

\subsubsection{Unsteady discharge}

This configuration can be representative of a main active branch of a braided river that laterally expands during a flood event (Zolezzi et al., 2012). To predict the temporal variability of the active (i.e. sediment-transporting) channel width, we extend the validity of equilibrium regime formulae to instantaneous discharge values during a flood event. Despite being rather crude, this assumption can be justified by the need to use a simple, physics-based relationship in a first theoretical attempt of this type. In addition, better alternatives don't seem to be available at present, despite recent approaches to investigate planform evolution of river channel with self evolving banks (Parker et al., 2011). We therefore assume the channel width being instantaneously at equilibrium with the imposed flow hydrograph and according to Eq. (2) we write this relationship as:

$B(\tau)=\alpha_{\mathrm{n}} Q(\tau)^{n_{\mathrm{W}}} \quad \tau=\sigma_{T}^{*} \tau^{*}$

where $\tau$ denotes dimensionless time, $\tau^{*}$ dimensional time and $\sigma_{T}^{*}$ reciprocal of the flood event duration. In this configuration, Eq. (9) has been assumed to describe both channel narrowing and widening.

\subsection{Mathematical formulation}

The analysis refers to a straight channel with erodible bed and banks made of homogeneous non-cohesive sediment. The governing equations are the 2-D shallow water equation and the sediment continuity equation, which can be written in the following dimensionless form:

$$
\begin{aligned}
& \frac{\partial U}{\partial t}+U \frac{\partial U}{\partial s}+V \frac{\partial U}{\partial n}+\frac{\partial H}{\partial s}+\bar{\beta} \frac{\tau_{s}}{D}=0 \\
& \frac{\partial V}{\partial t}+U \frac{\partial V}{\partial s}+V \frac{\partial V}{\partial n}+\frac{\partial H}{\partial n}+\bar{\beta} \frac{\tau_{\mathrm{n}}}{D}=0 \\
& \frac{\partial D}{\partial t}+\frac{\partial U D}{\partial s}+\frac{\partial V D}{\partial n}=0 ; \\
& \frac{\partial}{\partial t}\left(F_{0}^{2} H-D\right)+Q_{0}\left(\frac{\partial Q_{\mathrm{s}}}{\partial s}+\frac{\partial Q_{\mathrm{n}}}{\partial n}\right)=0 .
\end{aligned}
$$

In Eqs. (10)-(13) classical scalings for theoretical river morphodynamics are employed: the longitudinal and transversal variables $s$ and $n$ are scaled by the half-channel width $\bar{B}_{0}^{*}$, chosen as reference, the averaged velocity components $(U, V)$, the water depth $D$, the free surface elevation $H$ and the shear stress are scaled using the reference state quantities $\left(\bar{U}_{0}^{*}, \bar{D}_{0}^{*}\right)$. The reference, basic state is defined through three dimensionless parameters: the mean width ratio $\bar{\beta}$, the mean relative roughness $\bar{d}_{\mathrm{s}}$ and the mean Shields parameter $\bar{\theta}$, which read:

$\bar{\beta}=\frac{\bar{B}_{0}^{*}}{\bar{D}_{0}^{*}} \quad \bar{d}_{\mathrm{s}}=\frac{d_{\mathrm{s}}^{*}}{\bar{D}_{0}^{*}} \quad \bar{\theta}=\frac{S}{\Delta \bar{d}_{\mathrm{s}}}$ 


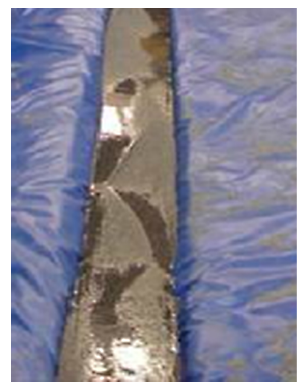

(a)

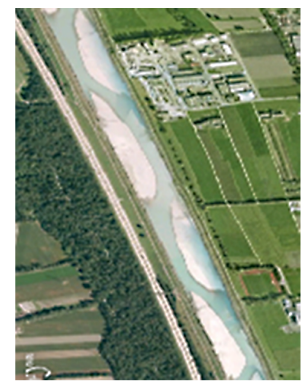

(b)

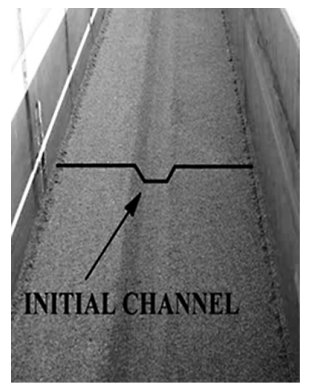

(c)

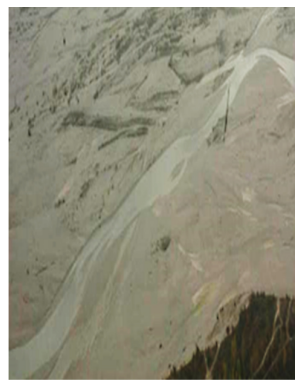

(d)

Fig. 1. Examples of channels belonging to the four reference configurations considered in the stability analysis. Each class presents different combinations of discharge and width unsteadiness. More in detail: (a) constant discharge and width (courtesy of Grecia A. Garcia Lugo); (b) variable discharge and fixed width, a reach of the Alpine Rhine in Switzerland; (c) erodible channel with unsteady width and constant discharge; (d) natural, laterally unconfined channel with unsteady width and discharge, Tagliamento River (NE Italy).

where $S$ is the longitudinal slope of the channel, $\Delta=\left(\rho_{\mathrm{s}} / \rho-\right.$ 1) the submerged sediment gravity and $d_{\mathrm{s}}^{*}$ the sediment diameter.

Boundary conditions in the lateral direction impose vanishing water and sediment flux orthogonal to the banks. When the active channel width changes in time, either because of bank erosion (constant discharge, Fig. 1c) or because of a combination of lateral inundation with erosional dynamics (variable discharge, Fig. 1d), the banklines are laterally moving at the timescale imposed by the process of temporal width variation (described through Eq. 20). Under those conditions, the vanishing lateral flux condition is assumed to apply at the instantaneous (moving) bank line position. Overall, the adopted approach results in neglecting the effect of sediment supply to the channel associated with lateral erosion on the process of bar stability. This seems reasonable given this first theoretical attempt although investigation of the actual role played by that effect will deserve attention in the future.

\subsection{Perturbation solution}

We solve the governing differential problem through a nonlinear, two-parameters perturbation approach. We then investigate under which conditions the reference uniform basic flow is unstable with respect to infinitesimal and sinusoidal perturbations of the bed elevation and of the other relevant flow quantities. The following two-parameters perturbation expansion, say for the water depth $D$, is adopted:

$$
\begin{aligned}
D & =1+\epsilon A(t)\left[\left(S_{\mathrm{m}} d_{10}\right) E_{1}(s, t)+c . c .\right]+\delta d_{01}(\tau) \\
& +\epsilon \delta\left[A(t) S_{\mathrm{m}} d_{11} E_{1}(s, t) d_{01}(\tau)+c . c .\right]+O\left(\epsilon^{2}, \delta^{2}\right)
\end{aligned}
$$

where $c . c$. denotes the complex conjugate, $\epsilon$ and $\delta$ are small parameters related to the amplitude of free bars and to the rate of width unsteadiness respectively. Moreover:

$$
\begin{aligned}
S_{\mathrm{m}} & =\sin \left(\frac{\pi}{2} m n\right) \quad C_{m}=\cos \left(\frac{\pi}{2} m n\right) \quad m=1,2, \ldots \\
E_{1} & =\exp (i \lambda s)
\end{aligned}
$$

where $\lambda$ is the dimensionless bar wavenumber.

By substituting the structure of Eq. (15) into Eqs. (10)(13), the original differential system is transformed into a series of linear homogeneous algebraic systems, at each order of approximation comparing in Eq. (15). The key property of this two-parameters perturbation expansion is that the $O(\epsilon \delta)$ is the lowest at which the spatial pattern of free bars is reproduced, because the solution at the order $O(\delta)$ is a perturbation of the reference basic flow and thus it is spatially uniform. The details of the solution procedure at the different orders of approximations are reported below.

\subsection{1 $O(\epsilon)$ : classical bar stability under conditions of constant width and discharge}

By substituting Eq. (15) into Eqs. (10)-(13) and collecting all terms at the leading order $\epsilon$, the classical linear free bar stability is recovered. This requires solving the following linear system:

$\mathbf{L}_{10}\left(\begin{array}{c}u_{10} \\ v_{10} \\ h_{10} \\ d_{10}\end{array}\right)=\left(\begin{array}{l}0 \\ 0 \\ 0 \\ 0\end{array}\right)$

where the linear differential operator $\mathbf{L}_{10}$ is reported, in its extended form, in Appendix A. Solution of Eq. (18) reveals that the amplitude of bars behaves exponentially in time:

$A(t)=\exp \left(\Omega_{10} t\right)$

with $\Omega_{10}=\Omega_{10, \mathrm{R}}+i \Omega_{10, I}, \Omega_{10, \mathrm{R}}$ bars growth rate and $\Omega_{10, I}$ bars angular frequency. Solution of Eq. (18) for the unknowns $u_{10}, v_{10}, h_{10}$ and $d_{10}$ with the parameters $\Omega, \lambda, \bar{\beta}, \bar{\theta}$ 
and $\bar{d}_{\mathrm{s}}$ requires a solvability condition, which allows calculation of the growth rate of bars and their angular frequency, for a given combination of bar wavenumber $\lambda$, the unperturbed width ratio $\bar{\beta}$, Shields parameter $\bar{\theta}$ and relative roughness $\bar{d}_{\mathrm{s}}$.

\subsection{2 $O(\delta)$ : linear correction to the basic flow related to width unsteadiness}

If the length of the channel is much shorter than the typical length of a flood wave, the temporal variability of channel width can be modelled as a temporal sequence of instantaneously uniform flows. To fit within the perturbation scheme, the adopted empirical relations for width unsteadiness are expanded in power of the small parameter $\delta$, which takes slightly different meanings depending on the considered hydromorphological configuration. In both the examined configurations (c) and (d) in Fig. 1 the perturbed channel width is then written in the form:

$B(\tau)=1+\delta b_{01}(\tau) \quad \delta<<1$

where $b_{01}$ is described through different functional expressions in the two cases, as it occurs for $\delta$. More specifically, for initially overnarrow channels subject to constant discharge and erodible banks:

$b_{01}(\tau)=-e^{-\tau} \quad \delta=\frac{B_{\mathrm{E}}^{*}-B_{0}^{*}}{B_{\mathrm{E}}^{*}}$,

while for the flood event over a laterally unconfined channels it holds:

$$
\begin{aligned}
b_{01}(\tau) & =\alpha_{\mathrm{n}} n_{\mathrm{B}} q_{01}(\tau) \quad Q_{0}(\tau)=1+\delta q_{01}(\tau) \\
\delta & =\frac{Q_{\max }^{*}-Q_{0}^{*}}{Q_{0}^{*}} .
\end{aligned}
$$

Here the unsteady discharge term $q_{01}$ is assigned as input data and it represents the functional shape of the given flow hydrograph.

According to the adopted perturbation approach, an analytical solution expressing the correction to the reference uniform flow due to width unsteadiness is obtained for the two configurations, (c) and (d). Therefore the unsteadinesscorrected basic flow reads:

$U_{0}=1+\delta u_{01}(\tau)+O\left(\delta^{2}\right)$,

$D_{0}=1+\delta d_{01}(\tau)+O\left(\delta^{2}\right)$.

By feeding the expansion (Eq. 23) into the governing Eqs. (10-13) we find for the unsteady discharge configuration $(d)$ :

$d_{01}(\tau)=p(1-\alpha) q_{01}(\tau)$,

$u_{01}(\tau)=(1-p)(1-\alpha) q_{01}(\tau)$

and, for steady discharge configuration (c):

$d_{01}(\tau)=-p b_{01}(\tau)$,

$u_{01}(\tau)=-(1-p) b_{01}(\tau)$.
In the above expressions the parameters $p$ and $\alpha$ take the value:

$$
p=\frac{2}{3-C_{\mathrm{D}}} \quad \alpha=\alpha_{\mathrm{n}} n_{\mathrm{B}} .
$$

\subsection{3 $O(\epsilon \delta)$ : effect of width variations on free bar stability}

By considering that the solution at the order $\delta$ is not dependent on the longitudinal variable $s$, the smallest order at which the spatial dependence of the fundamental is reproduced is the order $\epsilon \delta$ (Eq. 15). At this order, therefore, the solution accounts for the effect of the temporal width variation on free bar instability. Using the mathematical operator $\mathbf{L}_{10}$ introduced in Eq. (18), at the order $\epsilon \delta$ the governing differential system reads:

$$
\begin{gathered}
\mathbf{L}_{10}\left(\begin{array}{c}
u_{11} \\
v_{11} \\
h_{11} \\
d_{11}
\end{array}\right)=\left(\begin{array}{c}
-a_{11}^{1} u_{10}-a_{14}^{1} d_{10} \\
-a_{22}^{1} v_{10} \\
-a_{31}^{1} u_{10}-a_{32}^{1} v_{10}-a_{34}^{1} d_{10} \\
i \Omega_{11} K_{1}+\left(-i \Omega_{10}\right) K_{2}-Q_{0} \Phi_{0} K_{3}
\end{array}\right) ; \\
K_{1}=\left(F_{0}^{2} h_{10}-d_{10}\right), K_{2}=K_{1} \Phi_{T}\left[2 u_{01}(\tau)+c_{D} d_{01}(\tau)\right], \\
K_{3}=a_{41}^{1} u_{10}+a_{42}^{1} v_{10}+a_{43}^{1} h_{10}+a_{44}^{1} d_{10},
\end{gathered}
$$

where $\mathbf{L}_{10}$ is the same linear algebraic operator found at the leading $O(\epsilon)$ and the coefficients $a_{i j}^{1}, K_{i}$ are related to the nonlinear, unsteady effect arising from the interaction between the fundamental perturbation $(\epsilon)$ and the unsteady correction to the basic flow due to width variability $(\delta)$. The expression of $a_{i j}^{1}, K_{i}$ are reported in Appendix A for the sake of brevity.

As for the leading order, since the determinant of $\mathbf{L}_{10}$ vanishes, a solvability (eigenrelation) analogous to that occurring at the leading order holds:

$f\left(\Omega_{11}, \lambda ; \Omega_{10}, \bar{\beta}, \bar{\theta}, \bar{d}_{\mathrm{s}}\right)=0$,

which allows to compute the correction $\Omega_{11}=\Omega_{11, \mathrm{R}}+$ $i \Omega_{11, I}$ to bar growth rate and angular frequency at the order $\epsilon \delta$. As it can be easily seen from Eqs. (28) and (29), the solution of the system (Eq. 28) depends on the solution found at the previous order $O(\epsilon)$, so the problem is solved in cascade. The corrected expression of the complex number $\Omega$ accounting for width unsteadiness is therefore:

$\Omega(\tau)=\Omega_{10}+\delta \Omega_{11}\left(\Omega_{10}\right) b_{01}(\tau)$

where $\Omega_{10}$ and $\Omega_{11}$ account respectively for the growth rate associated to the fundamental perturbation and for the component related to the unsteadiness due to channel width variability.

\section{Results}

Results obtained by performing the analysis described above are here reported for the two analyzed morphological configurations: a laterally unconfined channel during a flood event 

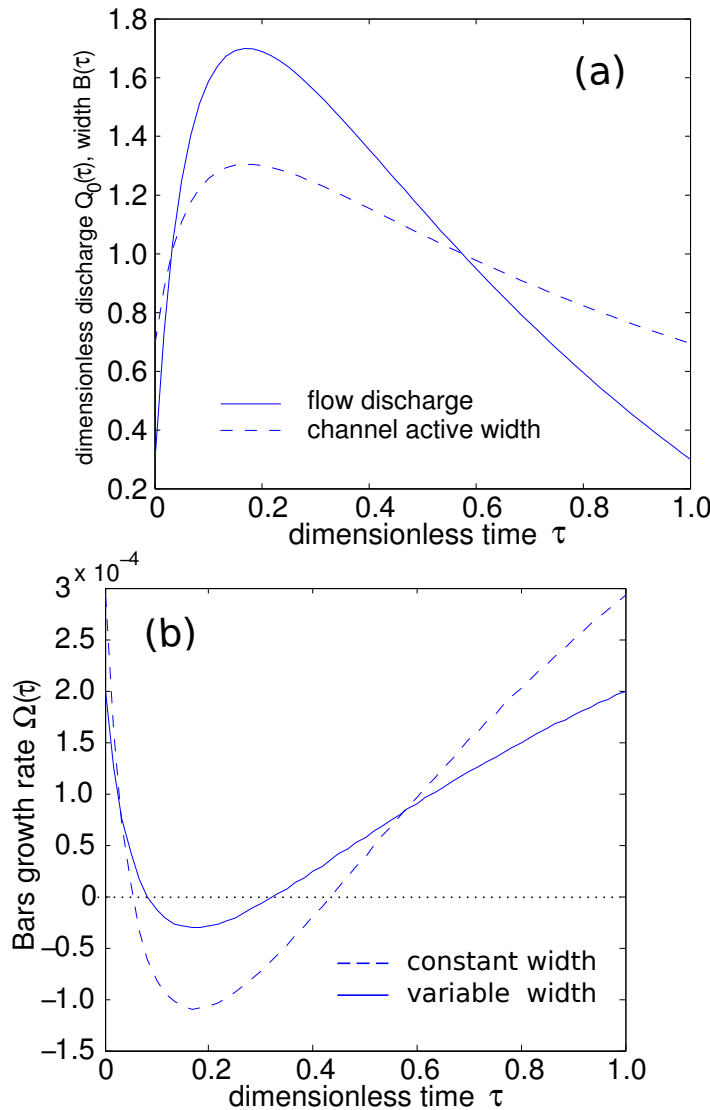

Fig. 2. (a) Temporal variability of the dimensionless discharge $Q_{0}(\tau)$ and associated temporal variability of the dimensionless channel width $B(\tau)$. (b) Values of the total bar growth rate $\Omega(\tau)$ for the flow event in (a) for laterally confined (dashed line) and unconfined (solid line) channels.

(unsteady discharge and width) and widening of an initially overnarrow channel under constant discharge.

\subsection{Unsteady discharge and variable width}

Figure 2 refers to the case of a flood event that occurs in a laterally unconfined channel. Figure 2 shows the dimensionless hydrograph of the flood event $Q_{0}(\tau)$ together with the linearized temporal variation of channel width, expressed by Eq. (20). The reference flow (basic state) has been assumed as the uniform flow with constant width occurring for the value $Q_{0}=1$.

As already pointed out by Tubino (1991) free bars under unsteady flow conditions are more stable during the rising and peak stage of the hydrograph, because the aspect ratio $\beta$ is decreasing and the critical value for bar formation $\beta_{\mathrm{c}}$ is increasing. In a channel with constant width the decrease of $\beta$ during the rising stage of the flood is related to decrease in water depth, while the increase of $\beta_{\mathrm{c}}$ is mainly associated with the increase in the Shields stress. On the contrary, bars
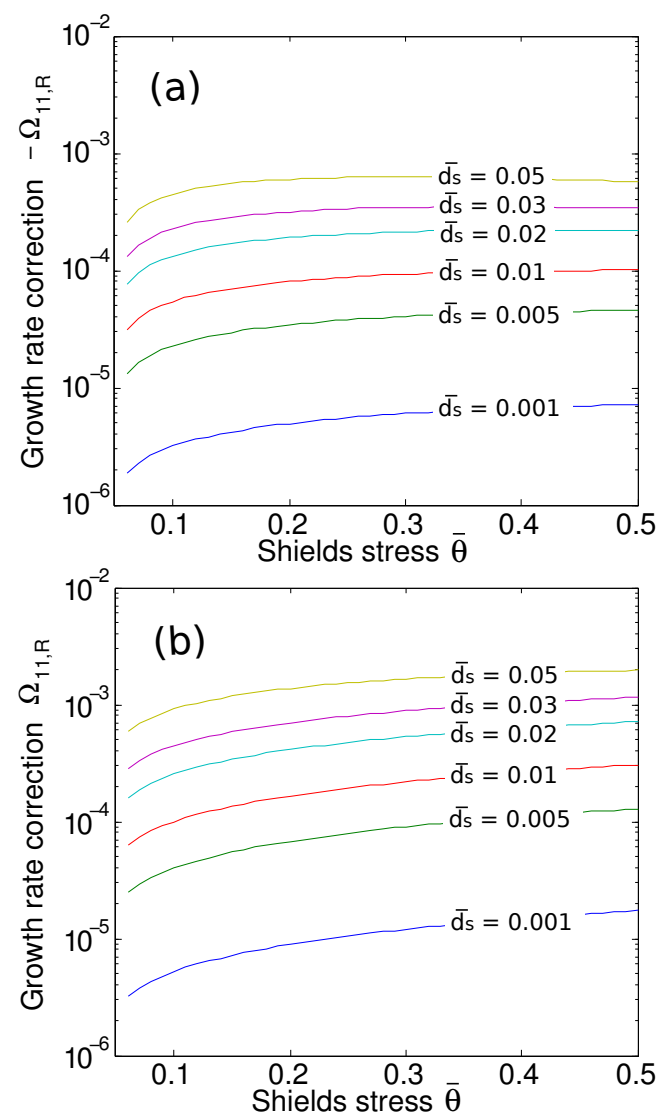

Fig. 3. The linear growth rate of alternate bars (real part of $\Omega_{11}$ ) is plotted vs. the Shields stress $\bar{\theta}$ for different values of the roughness parameter $\bar{d}_{\mathrm{s}}$ for the case of variable width with (a) variable, (b) constant discharge.

are more likely to form during the decreasing phase where the value of $\beta$ increases against a decreasing $\beta_{\mathrm{c}}$ value.

Figure 2 shows that accounting for temporal width variability results in damping the stabilizing effect associated with flow unsteadiness in a channel where the width does not change in time. Namely, when discharge exceeds the reference flow value $\left(Q_{0}(\tau)>1\right)$ the reduction in the bar growth rate is less pronounced than in the case with constant width.

As the temporal behavior of the complete solution is fundamentally controlled by the structure assigned to the temporal variable $q_{01}$ or $b_{01}$, it is informative to focus on the contribution given by the unsteady term $\Omega_{11, \mathrm{R}}$, which is invariably negative, as reported in Fig. 3a.

\subsection{Constant discharge and variable width}

Application of the bar theory with reference flow parameters that instantaneously adapt to the evolving width value indicate that free bars tend to form during the widening process because the value of the aspect ratio $\beta$ increases, while the critical value $\beta_{\mathrm{c}}$ is decreasing, being $\beta_{\mathrm{c}}$ a growing function of the Shields stress, which decreases with widening. 
Moreover, results obtained by applying the present theoretical analysis to an initially overnarrow channel, characterized by erodible banks and constant discharge, reveals a net positive contribution of the width unsteadiness to bar growth rate, $\Omega_{11, \mathrm{R}}>0$ (Fig. 3b). Channel widening is therefore predicted to enhance bar instability compared to the configuration with constant width. Moreover, as it can be recognized from Eq. (21), this effect is stronger when the difference between the initially imposed channel width and the equilibrium width is higher (Fig. 4). Width unsteadiness may therefore determine a complete reversal of bar stability conditions compared to the constant width case. This behaviour is related to the continuity equation for the fluid phase: in channels with constant discharge, the widening process is associated with a decrease of the cross-sectional averaged velocity and depth (Eq. 26), while in channels with variable discharge the temporal width variability is driven by the flood hydrograph: therefore widening during the rising stage is related to a contemporary increase in both cross-sectional averaged velocity and depth (Eq. 25). Such continuity effect mathematically determines opposite signs of the term $b_{01}$ in the two configurations, which are then reflected in the opposite signs of $\Omega_{11, \mathrm{R}}$ (Fig. 3a and $\mathrm{b}$ ).

More general results can be presented by examining the dependence of the most unstable wavelength at the initial time on the Shields stress $\theta$ and on the relative roughness $d_{\mathrm{s}}$ (Fig. 4b). This was achieved by assigning as reference state (equilibrium) a wide enough channel to guarantee barforming condition at the initial stage of the erosion process, where, the width is smaller. Shorter bars are therefore promoted, as the most unstable wavenumber is larger. Theoretical predictions of instability enhancement of shorter bars are in qualitative agreement with the experimental observations of Bertoldi and Tubino (2005).

\section{Discussion}

A novel stability analysis for free bars in channels with temporally variable channel width has been developed. Simple relationships describing the variability of the active channel in time allow us to develop a two-parameters perturbation expansion able to quantify the role of width unsteadiness by the interaction of the fundamental bar perturbation with the correction of the basic flow associated with the widening/narrowing processes. Despite the adopted simplifying hypothesis to describe the widening process in rivers channel, the method developed allows a first quantitative investigation of bars dynamics in laterally unconfined channels. Considering that little has been done in literature to investigate the effect of temporal width adjustments (see Parker et al., 2011), another novel point of the work consists in the derivation of a physically-based relationship for width variability under both steady and unsteady discharge conditons. The suitability of the adopted approach finds qualitative sup-
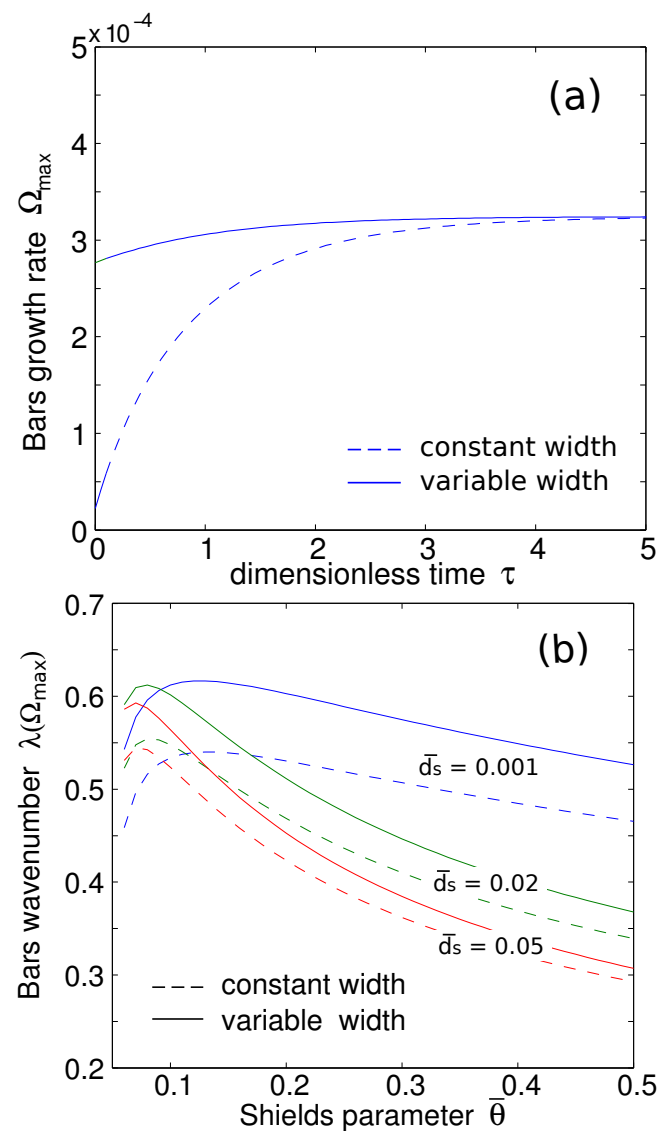

Fig. 4. Comparison between values predicted by applying the present theory (solid line) and the classical linear bar theory applied by assuming steady flow at each temporal step (dashed line). (a) shows difference in maximum bar growth rate and (b) shows the values of the most unstable bar wavenumber plotted against the Shields parameter $\bar{\theta}$ for different value of the roughness parameter $\bar{d}_{\mathrm{S}}$ at the initial time of the process.

port in the experiments of Bertoldi and Tubino (2005) and of Visconti et al. (2010), who specifically mentioned that in the initial stage of a run, bank erosion increases the channel width keeping the channel straight. The assumption of width variation to be small (say initial stage of laboratory experiment) seems to be reasonable in the light of this. Moreover, data provided from laboratory experiments can be used to validate the relaxation relation here proposed as well as the related time scale adopted.

Results obtained show, curiously, two formally opposite behaviours for the unsteady term $\Omega_{11, \mathrm{R}}$ when considering channel width variability. The sign of $\Omega_{11, \mathrm{R}}$ is controlled in a rather complex way by the variables defining the basic flow: $\beta, \theta, d s$ and the growth rate computed for the fundamental perturbation $\Omega_{10, R}$ as reported in Eq. (29). Namely, in the case of unsteady discharge this term is found to be negative, while in the other case it gives a positive contribution to bars growth rate. Moreover, during the rising and peak 
stage of a flood in a laterally unconfined channel, bar stability is increased, thus suggesting a non-trivial effect of width unsteadiness. In association to this destabilizing action, the most unstable bars present a shorter length compared to that predicted by the same theory developed for steady channel width.

Overall, theoretical predictions are in qualitative agreement with both the field observations of Zolezzi et al. (2012) and the experimental observation of (Bertoldi and Tubino, 2005). Further research is needed to apply the present theory to real cases, both in the field and in the laboratory, to assess to which extent the present theory can actually provide a relevant step towards an increased applicability of bar theories to complex channel geometries. Further research shall also concentrate to derive physically-based relationships for channel width variability.

Acknowledgements. This work has been partially carried out within the SMART Joint Doctorate (Science for the MAnagement of Rivers and their Tidal systems) supported by the Erasmus Mundus programme of the European Union. The authors are grateful to Brad Murray and the anonymous reviewer for their suggestions, which were useful in improving the paper.

\section{References}

Ashmore, P.: Laboratory modelling of gravel braided stream morphology. Earth Surf. Process. Land., 7, 201-225, 1982.

Ashmore, P.: How do gravel-bed rivers braid? Can. J. Earth Sci., 28, 326-341, 1991.

Bertoldi, W. and Tubino, M.: Bed and bank evolution of bifurcating channels, Water Resour. Res., 41, W07001, doi:10.1029/2004WR003333, 2005.

Church, M. and Rice, S. P.: Form and growth of bars in a wandering gravel-bed river, Earth Surf. Process. Land., 34, 1422-1432, 2009.

Colombini, M., Seminara, G., and Tubino, M.: Finite-amplitude alternate bars, J. Fluid Mech., 181, 213-232, 1987.
Crosato, A. and Mosselman, E.: Simple physics-based predictor for the number of river bars and the transition between meandering and braiding. Water Resour. Res., 45, W03424, doi:10.1029/2008WR007242, 2009.

Hall, P.: Alternating bar instabilities in unsteady channel flows over erodible beds, J. Fluid Mech., 499, 49-73, doi:10.1017/S0022112003006219, 2004.

Lewin, J.: Initiation of bed forms and meanders in coarse grained sediment. Geolog. Soc. Am. Bull., 87, 281-285, 1976.

Parker, G.: On the cause and characteristic scales of meandering and braiding in rivers, J. Fluid Mech., 76, 457-479, doi:10.1017/S0022112076000748, 1976.

Parker, G., Shimizu, Y., Wilkerson, G. V., Eke, E. C., Abad, J.D., Lauer, J. W., Paola, C., Dietrich, W. E., and Voller, V. R.: A new framework for modeling the migration of meandering rivers. Earth Surf. Process. Land., 36, 70-86, doi:10.1002/esp.2113, 2011.

Parker, G., Wilcock, P. R., Paola, C., Dietrich, W. E., and Pitlick, J.: Physical basis for quasi-universal relations describing bankfull hydraulic geometry of single-thread gravel bed rivers, J. Geophys. Res., 112, F04005, doi:10.1029/2006JF000549, 2007.

Repetto, R., Tubino, M., and Paola, C.: Planimetric instability of channels with variable width, J. Fluid Mech., 457, 79-109, 2002.

Surian, N. and Rinaldi, M.: Morphological response to river engineering and management in alluvial channels in italy, Geomorphology, 50, 307-326, 2003.

Tubino, M.: Growth of alternate bars in unsteady flow, Water Resour. Res., 27, 37-52, 1991.

Visconti, F., Camporeale, C., and Ridolfi, L.: Role of discharge variability on pseudomeandering channel morphodynamics: Results from laboratory experiments, J. Geophys. Res., 115, F04042, doi:10.1029/2010JF001742, 2010.

Welford, M.: A field-test of Tubino's (1991) model of alternate bar formation, Earth Surf. Process. Land., 19, 287-297, doi:10.1002/esp.3290190402, 1994.

Zolezzi, G., Bertoldi, W., and Tubino, M.: Morphodynamics of bars in gravel-bed rivers: Bridging analytical models and field observations, in Gravel-bed Rivers: Processes, Tools, Environments, edited by Church, M., Biron, P. M., and Roy, A., Chap. 6, 69-89, John Wiley \& Sons, Chichester, UK, 2012. 


\section{Appendix A}

Coefficients of the linear system at the order $O(\epsilon)$ and $\boldsymbol{O}(\boldsymbol{\epsilon} \boldsymbol{\delta})$

Collecting the terms at the order $(\epsilon)$ and $(\epsilon \delta)$ in the system

$$
\begin{gathered}
a_{i 1}(t) u_{1}+a_{i 2}(t) v_{1}+a_{i 3}(t) h_{1}+a_{i 4}(t) d_{1}=0, \\
i=1,2,3,4
\end{gathered}
$$

the coefficients $a_{i, j}(t)$ can be expaned as

$a_{i j}(t)=a_{i j}^{0}+a_{i j}^{1}(t) \quad i, j=1,2,3,4$

and for the specific case they read as follow:

$$
\begin{aligned}
& a_{11}^{0}=i \lambda+2 \beta_{0} C_{0} \\
& \quad a_{11}^{1}(t)=a_{11}^{0} u_{01}(t)+\left[2 \beta_{0} C_{0}\left(C_{\mathrm{D}}-1\right)\right] d_{01}(t) ; \\
& a_{13}^{0}=i \lambda \\
& \quad a_{13}^{1}(t)=0 ; \\
& a_{14}^{0}=\beta_{0} C_{0}\left(C_{\mathrm{D}}-1\right) \\
& \quad a_{14}^{1}(t)=\left[2 \beta_{0} C_{0}\left(C_{\mathrm{D}}-1\right)\right] u_{01}(t)+ \\
& \quad \beta_{0} C_{0}\left(P_{11}+C_{\mathrm{D}}\left(C_{\mathrm{D}}-1\right)+2\right] d_{01}(t) ; \\
& a_{22}^{0}=i \lambda+\beta_{0} C_{0} \\
& \quad a_{22}^{1}(t)=\left[i \lambda+\beta_{0} C_{0}\right] u_{01}(t)+\beta_{0} C_{0}\left(C_{\mathrm{D}}-1\right) d_{01}(t) ; \\
& a_{23}^{0}=\frac{\pi}{2} \\
& \quad a_{23}^{1}(t)=0 ; \\
& a_{31}^{0}=i \lambda \\
& \quad a_{31}^{1}(t)=a_{31}^{0} d_{01}(t) ; \\
& a_{32}^{0}=-\frac{\pi}{2} \\
& \quad a_{32}^{1}(t)=a_{32}^{0} d_{01}(t) ; \\
& a_{34}^{0}=i \lambda \\
& \quad a_{34}^{1}(t)=a_{34}^{0} u_{01}(t) ; \\
& a_{41}^{0}=2 i \lambda \Phi_{T} \\
& \quad a_{41}^{1}(t)=\left[2 i \lambda\left(P_{21 \mathrm{~F}}-\Phi_{T}\right)\right] u_{01}(t)+2 i \lambda P_{21 \mathrm{D}} d_{01}(t) ; \\
& a_{42}^{0}=-\frac{\pi}{2} \\
& \quad a_{42}^{1}(t)=-a_{42}^{0} u_{01}(t) ; \\
& a_{43}^{0}=\frac{\pi^{2} r F_{0}^{2}}{4 \beta_{0} \sqrt{\theta_{0}}} \\
& \quad a_{43}^{1}(t)=-a_{43}^{0} u_{01}(t)+\left[-\frac{C_{\mathrm{D}}}{2} a_{43}^{0}\right] d_{01}(t) ; \\
& a_{44}^{0}=-\frac{\pi^{2} r F_{0}^{2}}{4 \beta_{0} \sqrt{\theta_{0}}}+i \lambda \Phi_{T} C_{\mathrm{D}} \\
& \quad a_{44}^{1}(t)=\left[\frac{a_{43}^{0}}{F_{0}^{2}}+i \lambda C_{\mathrm{D}} P_{21 \mathrm{~F}}\right] u_{01}(t) \\
& \quad+\left[\frac{C_{\mathrm{D}} a_{43}^{0}}{2 F_{0}^{2}}+i \lambda\left(\Phi_{T} C_{\mathrm{D}} P_{11}+C_{\mathrm{D}} P_{21 \mathrm{D}}\right)\right] d_{01}(t), \\
& \quad
\end{aligned}
$$

having defined

$$
\begin{aligned}
& P_{11}=C_{\mathrm{D}}\left(\frac{C_{\mathrm{D}}}{2}-1\right), \\
& P_{21 \mathrm{~F}}=-2 \theta_{c} \Phi_{T}, \\
& P_{21 \mathrm{D}}=-C_{\mathrm{D}} \theta_{c} \Phi_{T}
\end{aligned}
$$

and

$$
\begin{aligned}
& C_{\mathrm{D}}=\frac{\left.C_{\mathrm{f}, \mathrm{D}}\right|_{0}}{C_{\mathrm{f} 0}}, \\
& \Phi_{T}=\left.\frac{\theta_{0}}{\Phi_{0}} \Phi_{, \theta}\right|_{\theta_{0}},
\end{aligned}
$$

where $C_{\mathrm{f}}$ denotes the friction coefficient, $\Phi$ the intensity of bedload transport and the subscript 0 refers to the reference state.

The system 18 for the order $O(\epsilon)$ is therefore written in the form

$$
\begin{gathered}
\left(\begin{array}{cccc}
a_{11}^{0} & 0 & a_{13}^{0} & a_{14}^{0} \\
0 & a_{22}^{0} & a_{23}^{0} & 0 \\
a_{31}^{0} & a_{32}^{0} & 0 & a_{34}^{0} \\
a_{41}^{0} Q_{o} \Phi_{o} & a_{42}^{0} Q_{o} \Phi_{0} & a_{43}^{0} Q_{0} \Phi_{o} F_{0}^{2}\left(-i \Omega_{10}\right) & a_{44}^{0} Q_{0} \Phi_{o} F_{0}^{2}\left(i \Omega_{10}\right)
\end{array}\right) \\
\left(\begin{array}{l}
u_{10} \\
v_{10} \\
h_{10} \\
d_{10}
\end{array}\right)=\left(\begin{array}{l}
0 \\
0 \\
0 \\
0
\end{array}\right) .
\end{gathered}
$$

The matrix of the system A6 has been represented in the text through the linear differential operator $\mathbf{L}_{10}$. 